\title{
Richmond line alliance: developing and delivering a complete rail system
}

\author{
P. Boulden \& M. Smith \\ AECOM, Australia
}

\begin{abstract}
This paper presents a case study of the Richmond Line Alliance in the development and delivery of a major project underpinning urban passenger rail growth in the Sydney metropolitan area. This paper demonstrates how rail engineers and designers can benefit from a holistic rail system's approach to project delivery through the practical application of an integrated project assurance philosophy executed from a technical, safety and quality perspective.

Advancing the topics of risk management and safety in transit systems, the paper uses the Richmond Line Duplication Stage 1 to present the results of outstanding industry practice and continuous improvement to conclude that:

- An incentivised commercial and delivery framework motivates execution;

- Application of interdisciplinary coordination processes expose conflicts and resolve issues of compatibility and consistency across design interfaces;

- Design validation and product assurance processes challenge the design team to consider the product from a wider perspective and ensure it is robust when tested by end user scenarios;

- A structured approach to design for constructability, operability, maintainability and safety can design out risk so far as reasonably practicable; and

- Project safety culture is enhanced by tools to personalise the safety message.
\end{abstract}

Keywords: rail systems, risk management, design validation, product assurance safety culture. 


\section{Introduction}

The Richmond Line Duplication Project is part of the NSW Government's Rail Clearways Program [1]. The project will provide capacity for additional peak services on the Richmond Branch Line to cater for future passenger demand [2]. The Richmond Line Duplication Stage 1 includes construction of an additional (duplicated) track between Quakers Hill and Schofields, together with a new station, as shown in Figure 1.

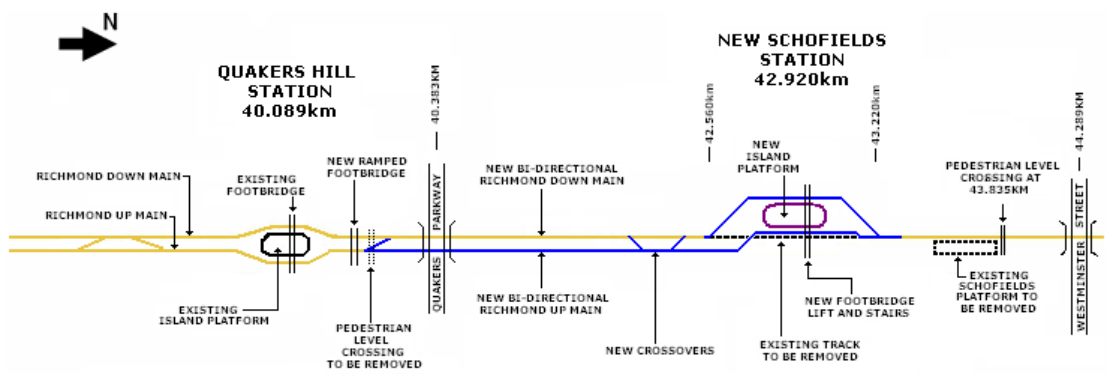

Figure 1: $\quad$ Richmond Line Duplication stage 1.

This paper uses the Richmond Line Duplication as a case study to demonstrate outstanding industry practice, continuous improvement and lessons learnt across the following facets of delivery:

- Interdisciplinary coordination and control;

- $\quad$ Project controls, design validation and product assurance;

- Value engineering;

- Designing for constructability, operability, maintainability and safety; and

- Project safety culture.

\section{Project delivery}

The Richmond Line Duplication Project is being delivered on behalf of the NSW Government by Transport Construction Authority (TCA) in an alliance known as the Richmond Line Alliance (RLA) comprising TCA, Leighton Contractors, AECOM, Sinclair Knight Merz, MVM Rail and Ansaldo STS [3]. The alliance brings together designers, architects and constructors into an integrated team operating under a common incentivised commercial and delivery framework.

As is typical in alliances, performance objectives were established as measurable Key Performance Indicators (KPIs) designed to govern and motivate all aspects of organisational behaviour and project execution.

In the case of the Richmond Line Alliance (RLA) the team were tasked to both define and execute project scope and in doing so were challenged to adopt the highest standards of project governance in delivering an assured end-product that was aligned fully with user requirements and met all engineering, design and implementation criteria that presented value to stakeholders. 


\subsection{Interdisciplinary coordination and control}

Front and centre of effective rail systems design is the key objective to ensure consistent Inter-Disciplinary Coordination (IDC) which accounts for the sometimes complex interaction between engineering disciplines and features in and around the rail corridor; both existing and new. The key to effective IDC is in the implementation of a simple and agile system that can track and flex with the volume and rate of change that naturally occurs, particularly during the early stages of the project while scope and requirements are being defined. For IDC to be effective, the process requires the complete buy-in of all design and construction functions as well as the client and project sponsor. It also requires an effective means of testing the designed outputs to expose conflicts or issues of compatibility and consistency across the many interfaces in the project [4].

In the case of the RLA, the vehicle for IDC was a series of workshops to review the latest designs. An inclusive and facilitated process was established involving the linear navigation of the rail corridor from beginning to end of the project scope. The value emerged as a result of the active discussions and determination of engineering interface solutions in and around pinch-points and conflicts and exposing data gaps and ambiguities. Issues were recorded on a simple spreadsheet and allocated to an appropriate owner to resolve. The process was iterated on a weekly basis with the key objective to repeat the reviews as more information, detail and certainty was injected into the design, while actively driving the issues count to zero.

The interface diagram in Figure 2 illustrates the key interfaces managed throughout design development.

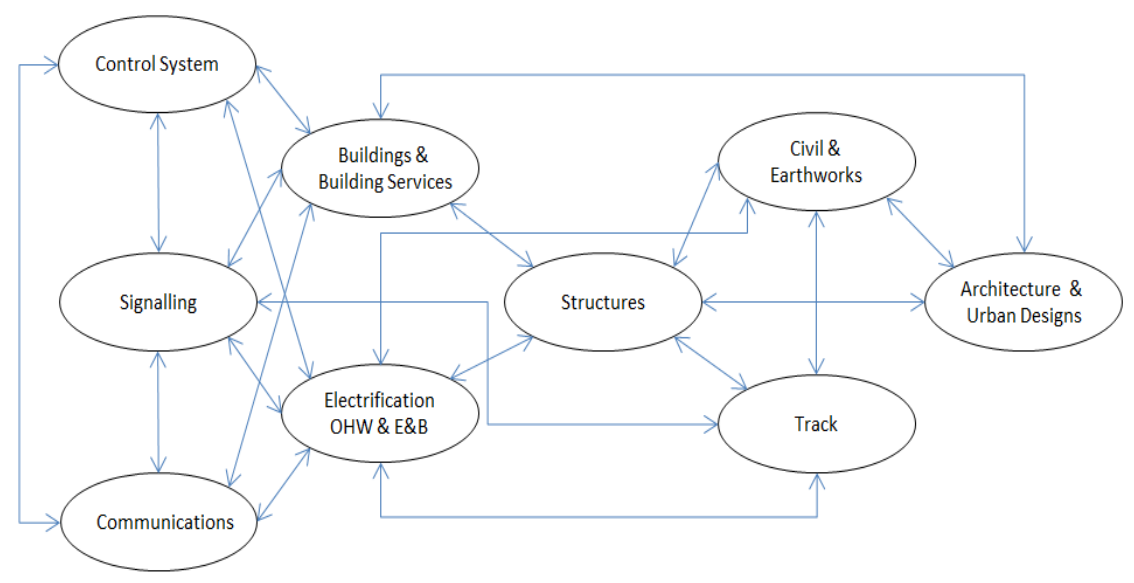

Figure 2: Design interface diagram.

A key feature of IDC involved engineering ownership of the interfaces with discipline specialists. This was realised by implementing a mandatory sign-off at each design submission stage whereby the lead designer was called upon to 
review and certify (as far as IDC goes) that the interfacing design was compliant and compatible. This collaborative cross-function sign-off process was fundamental to the consistent understanding and management of interface issues and implications. Culturally and behaviourally the process served to further reduce barriers between disciplines and actively encouraged a multi-disciplinary approach and attitude to support effective rail system definition.

The active IDC process was implemented to good effect within the RLA. Examples of tangible benefits resulting from this value-add process included:

- Locating the optimum position of the telecommunications building at Schofields station relative to the track, services and stage works;

- $\quad$ Providing overhead wiring and signal structure bases clear of utilities and drainage routes and assessing changes in locations during design development, as shown in Figure 3;

- Final route of HV aerial transmission line catering for pole positioning, earthing and bonding infrastructure and location of the cable to provide adequate clearance to existing and proposed buildings and structures in the area, as well as catering for the impact of a new public footpath and cycle way introduced as part of the project; and

- Optimum provision and location of underline crossings for the containment of signalling, communications, power and utility services - determining best fit volume type and positioning of these assets.

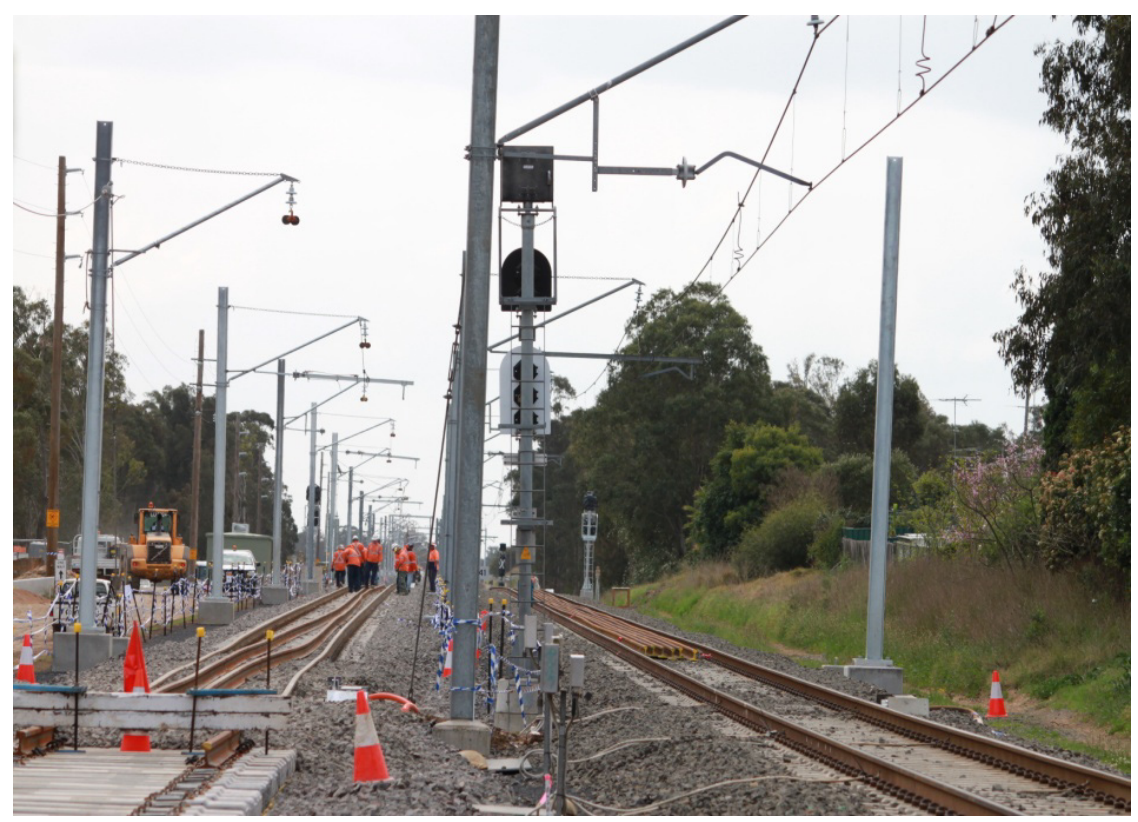

Figure 3: $\quad$ Example of multidisciplinary interfaces. 


\subsection{Project controls, design validation and product assurance}

\subsubsection{Project controls}

In the case of the RLA, the owner (RailCorp), the client (TCA) and the alliance itself established and operated a defined suite of processes within which project development, design and implementation were executed. The RLA integrated these processes into a single cohesive system which administered delivery to the client at all stages. Importantly, the RLA also resourced the Project Controls task with a team responsible for managing delivery and interface approvals throughout. Key to this success was the adoption of a production line approach recognising the sheer volume and effort to produce multiple related design packages for approval whilst ensuring stewardship for timely delivery. This process generated a total of 81 design packages in the 26 weeks from July 2009 to December 2009.

\subsubsection{Design validation}

The Richmond Line Duplication design progressed through distinct submission and approval stage-gates at pre-concept design, concept design, reference design, $30 \%$ detailed design, 70\% detailed design, 100\% detailed design and finally approved for construction. At all stages the owner executed a right of review of each design package. This ensured requirements and preferences were effectively articulated in the first instance. The process of capturing and actioning comments raised is substantial and occasionally complex, considering the inter-disciplinary interactions of change. Emerging or refined information of existing assets obtained from site can also require accommodation as the designer seeks to present the most accurate and latest design.

In the case of the RLA, a key critical success factor was the early recognition of the complexity and program style discipline required to manage client communications. While the mechanics were managed using standard tools of the trade such as spreadsheets and tracker programme reporting - the real differentiator was the effective buy-in of the RailCorp owner and approval body of the processes used by RLA to manage client communications. This required the consistent engagement of RailCorp's asset manager, operators, engineers and project management team. As well as presenting the design stages and effecting a project solution acceptable across a wide range of stakeholders, the project succeeded at a human level, with issues and comments being actively resolved through proactive interface at all levels, often onsite as shown in Figure 4 - with formal communications and protocol in support.

\subsubsection{Product assurance}

Modern project development and delivery recognises compliance to relevant standards or codes as only the first step towards the presentation of an assured product. By definition, this is characterised by an output that is robust, carries a high degree of certainty and provides reassurance to stakeholders and meets expectations. An example of an assured product on the RLA is shown in Figure 5. 


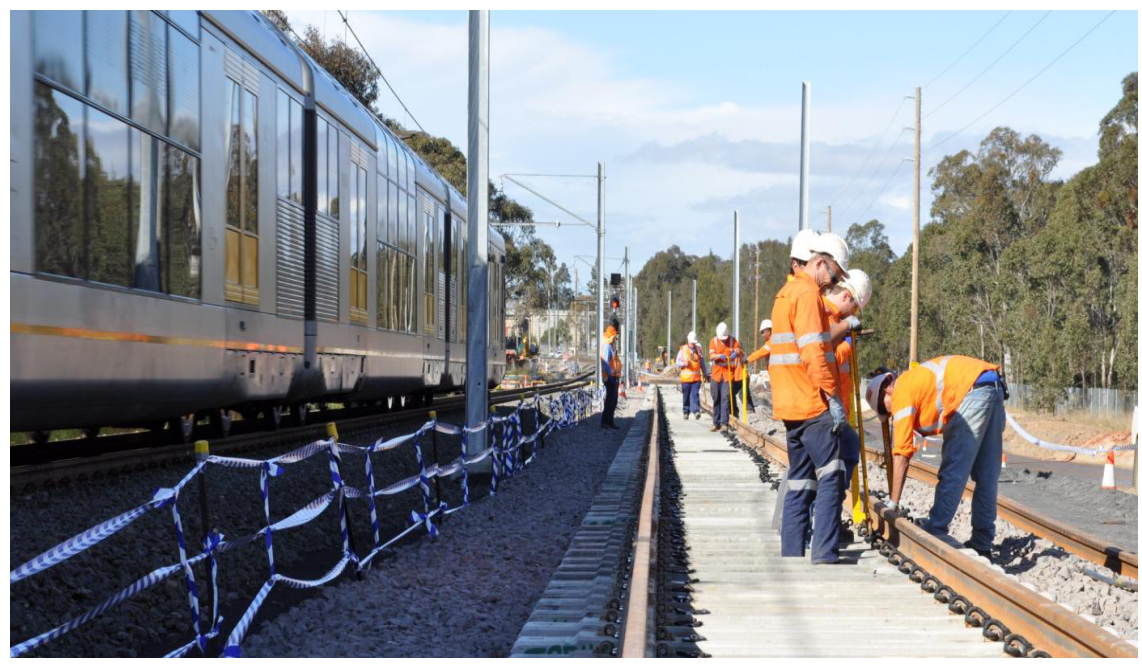

Figure 4: Validating the designed product.

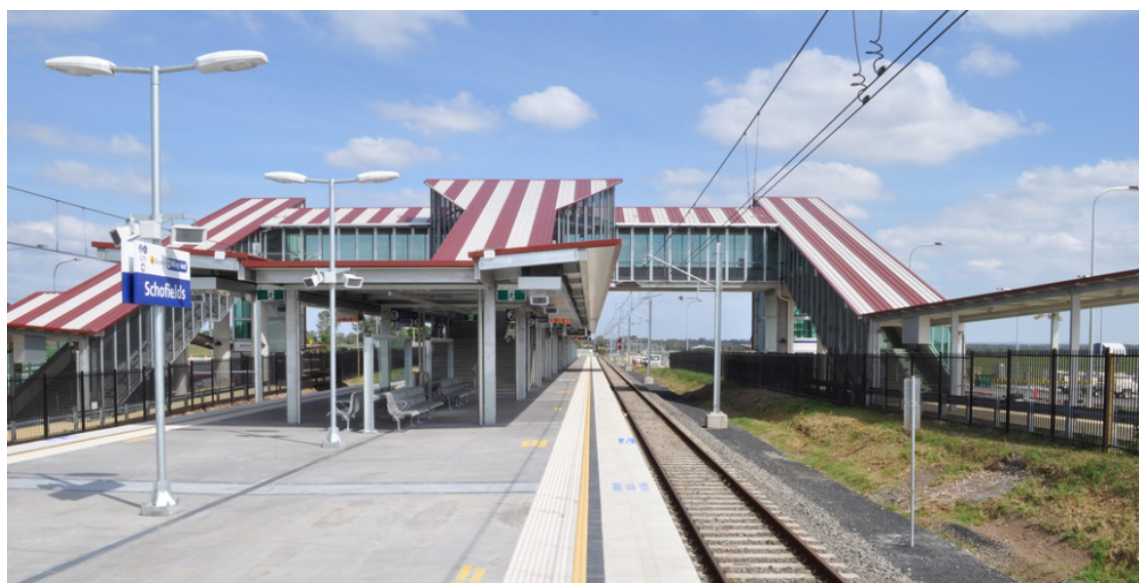

Figure 5: An assured product.

In the case of the RLA, the client, TCA deployed assurance engineers into the team whose role was to effectively verify and challenge the design outputs applying a layer of rigour to ensure the designed output was robust to go forward for owner's (RailCorp) consideration and approval. In addition, the RLA provided a suite of operational, technical and safety arguments in support of certain aspects of the design. This had the effect of challenging the design team to consider the designed product from a wider perspective and to ensure it was robust when tested by a whole host of end user scenarios and considerations. 
In the case of the RLA, this protocol leveraged particular benefits across the following examples.

- Maintenance Access Track: the RLA introduced a maintenance access route along the western edge of the rail corridor. This proved to be a complex exercise of accommodating the owner's access and maintenance requirements as well as negotiating a sufficient land envelope with neighbouring landowners as part of the land acquisition process. The complexities concerning the definition of this asset were eased through clear and consistent lines of communication with the operators and maintainers within RailCorp who were able to represent and interpret their user specifications, addressing aspects such as vehicular access (for maintenance and renewal), planned and emergency conditions, train driver access and egress. This enabled the TCA to conduct land negotiations confident that operational as well as engineering considerations were accounted for.

- New Schofields Station, as shown in Figure 6: early engagement with operational and assurance engineers proved invaluable in defining the envelope of this major asset and the consequential track alignment and land acquisition parameters. This process called for the engagement of RailCorp operators and station working group to develop all aspects of the station asset specification. Among the many issues tackled and resolved was the curvature of the alignment through the station itself. While within standards, the curve presented a concern and was tested by developing technical and safety arguments that assessed and presented risk, cost and value for both the curve and straight scenarios. The resulting analysis supported the original slightly curved platform and this was ultimately authorised by stakeholders as the preferred concept to be developed during detailed design.

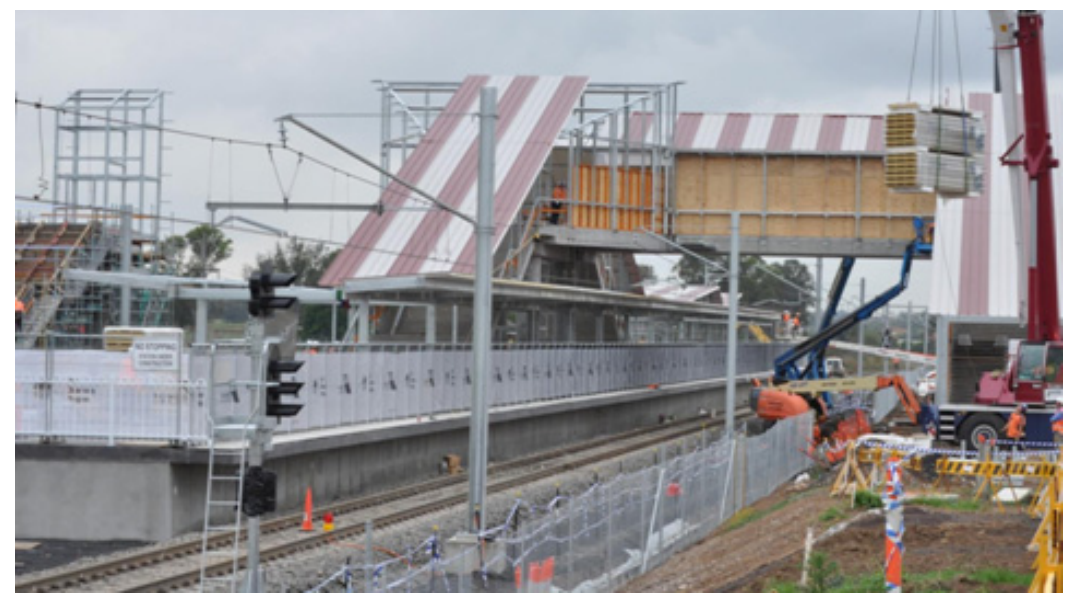

Figure 6: Schofields station. 


\subsection{Value engineering}

The RLA engaged Value Engineering (VE) effort from the earliest stages of project development. Operating as a separate work-stream within the alliance, VE was realised using staged and facilitated workshops involving designers, constructors, operators, project sponsors and client/owner stakeholders. The process involved the development of a shopping list of initiatives to increase value and/or reduce cost. This list was then iteratively reduced during focussed sessions as each initiative was tested by developed feasibility, value and cost reduction criteria. The key advantage the VE process centred on the early (preconcept) stage of engagement. This led to VE initiatives being adopted proactively into the scope and accounted for within the earliest iterations of the Target Outturn Cost (TOC). Examples of these include:

- Building $11 \mathrm{kV}$ capability in the Stage 1 duplication to Schofields to enable final commissioning of $11 \mathrm{kV}$ in the Stage 2 duplication to Vineyard;

- Installing the HV line underground within the combined services route in some areas, but aerial in others;

- Optimising the location of the traction power substation, as shown in Figure 7. During the early concept stages, this asset was located to the west of the rail corridor, requiring additional land acquisition. Subsequent reengineering resulted in relocation to the east of the corridor within a vacant lot. The key driver for this was to optimise the position relative to the rail corridor and minimise the land acquisition envelope and cost to the project; and

- Building a new rail underbridge at Schofields, as shown in Figure 8, to allow for a future underpass of the rail line by the roads agency during an extended track possession initially intended to commission the new track.

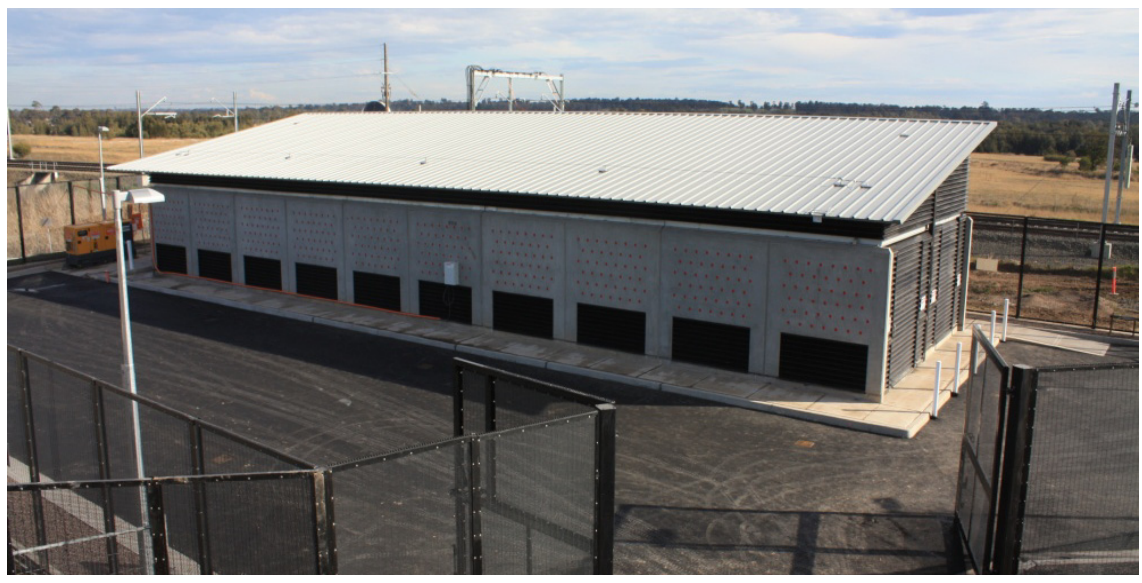

Figure 7: Traction substation location optimisation. 


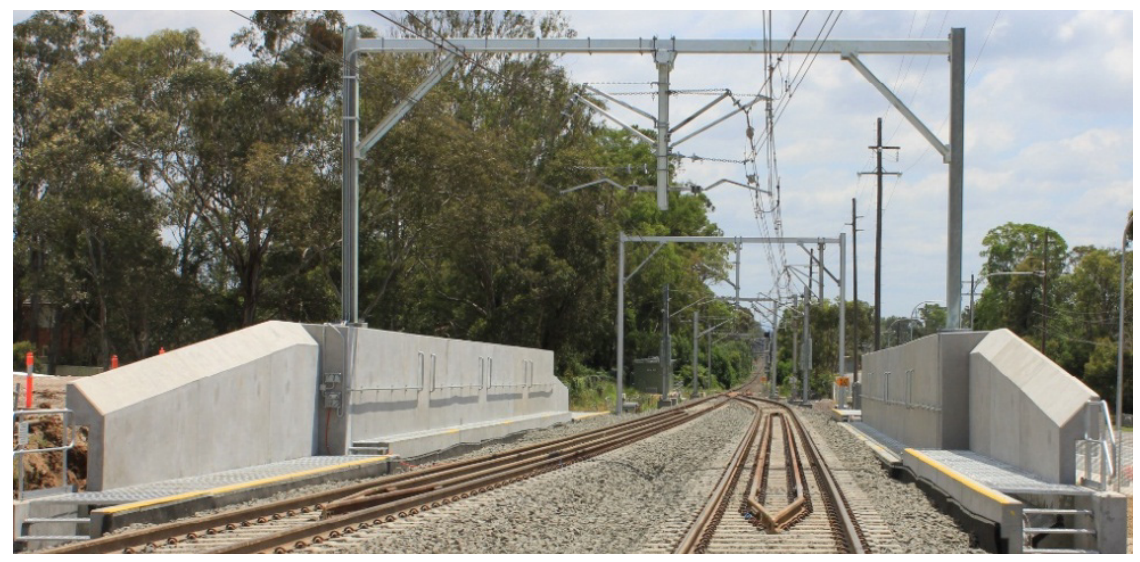

Figure 8: Underbridge for future road crossing.

\subsection{Designing for constructability, operability, maintainability and safety}

Working definitions of these aspects of design are as follows.

- Constructability: the ability to build the design in an optimum fashion within a set parameters including time, cost and track access.

- Operability: the degree to which the user can realise network functionality and train movements as defined for the project.

- Maintainability: The degree to which the completed project and asset configuration meets the owner's requirements in terms of access, asset life and performance. Issues such as access and the deployment of new or dissimilar technologies come into play here.

- Safety in Design: A concept that encourages designers, in particular, to design out risks during design development. The concept supports the view that along with quality and cost, safety is determined during design.

On RLA the design output at each stage was actively tested by each of these parameters, with reports generated as part of the submissions detailing the project response against each feature. Design development was characterised by engaging the client, owners and operators with designers and constructors to test the design against the terms of reference as specified at project definition. In the area of safety in design, the RLA deployed a number of client prescribed processes and tools such as preliminary hazard analysis and risk assessment and quantification of the designed product, as described by Welschen [5]. Of particular note was the degree to which the designers embraced and contributed to the process. RLA succeeded in bringing safety in design to life by deploying a structured 'risk identification - risk assessment - mitigation - allocation transfer' protocol which drove the design team to design out risk as far as is reasonably practicable. Once the process was exhausted the risk was either allocated or transferred as appropriate without being lost.

A good example of where RLA employed safety in design to benefit the project was in the determination of overhead wiring (OHW) structure locations 
and types, as shown in Figure 9. A number of OHW structures required to support the duplication were reviewed by the operator and maintainer, RailCorp, as part of an iterative hazard and risk review process. Where some structures were found to be in between existing and proposed track sections, the specific locations were re-engineered to relocate the structures and prevent the requirement for asset access between two live tracks.

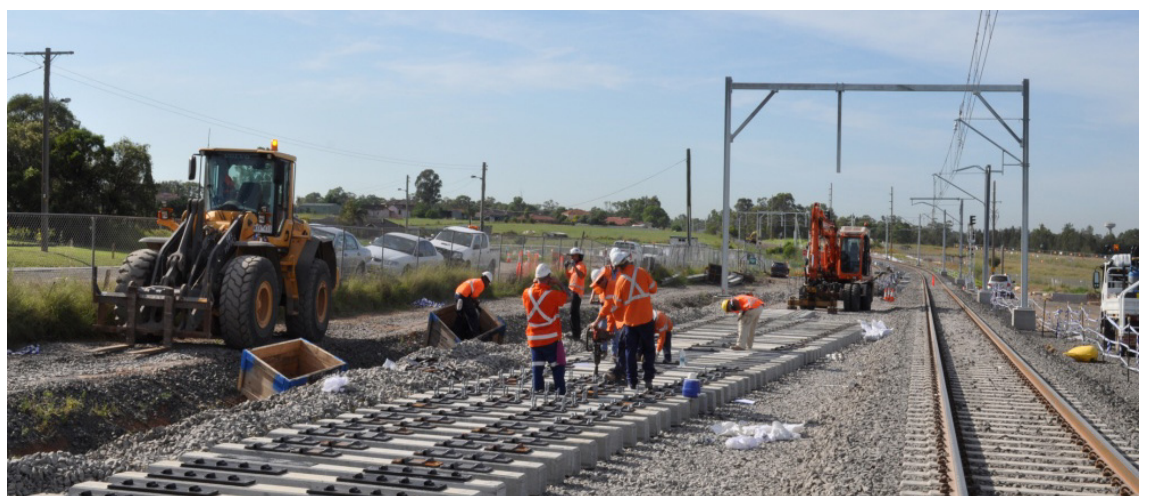

Figure 9: Design for safety in construction and during operation.

\subsection{Project safety culture}

The RLA led safety from the front during all stages of project development and delivery.

Developing, refining and publicising the principles governing behaviour and expectations were addressed initially. The safety culture program was extensively launched across all levels of the project using Safety Passports, as shown in Figure 10, as a tool to lead, engage and personalise the safety message to facilitate commitment. This cultural paradigm was developed and maintained through a concerted program of group and individual communications relating all aspects of work and life to the safety message underpinning the campaign.

From a designer's perspective, the RLA successfully created a culture whereby safety became a directly relevant parameter and aspect to the design task. Designers were effectively engaged to problem solve from a safety perspective; an activity hitherto principally the domain of the constructor.

This cultural stamp established by the RLA enabled safety in design to be a tangible and value-adding aspect of the designer's portfolio of deliverables and added a critical dimension of meaning and relevance to the earliest stages of design. This is the ideal point in the project lifecycle to inject this level of thinking and approach.

The project safety culture in the RLA acted as a positive enabler to ensure risks or safety considerations presented by the rail system to the client, owner, operator and the travelling public were given due consideration throughout each stage of design and construction. 


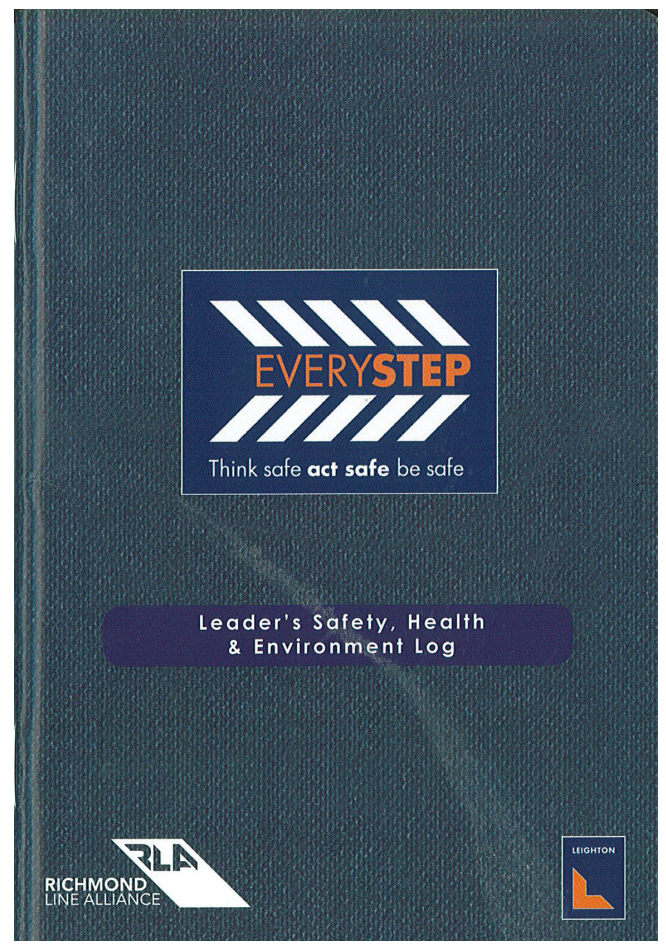

Figure 10: $\quad$ Personalising the safety culture.

\section{Conclusion}

The RLA successfully completed the design tasks between 2009 and early 2011 and constructed and commissioned the project between 2010 and late 2011.

Underpinning this performance was the uncompromising adoption of the processes described in this paper (among many others) as an integral aspect of alliance operation. In addition, a program and delivery discipline and focus permeated the team as a key value which was a key cornerstone of the delivery ethos from which RLA derived real benefit.

Considering the project delivered by the RLA, as demonstrated by the end product featured in Figure 11, it is logical to conclude that project performance benefits significantly from the adoption of processes as described in this paper. Furthermore, this reinforces the premise that successful project development and delivery is so much more than just technical design. This is a point of principle that owners, clients, constructors and designers alike should consider for future projects. 


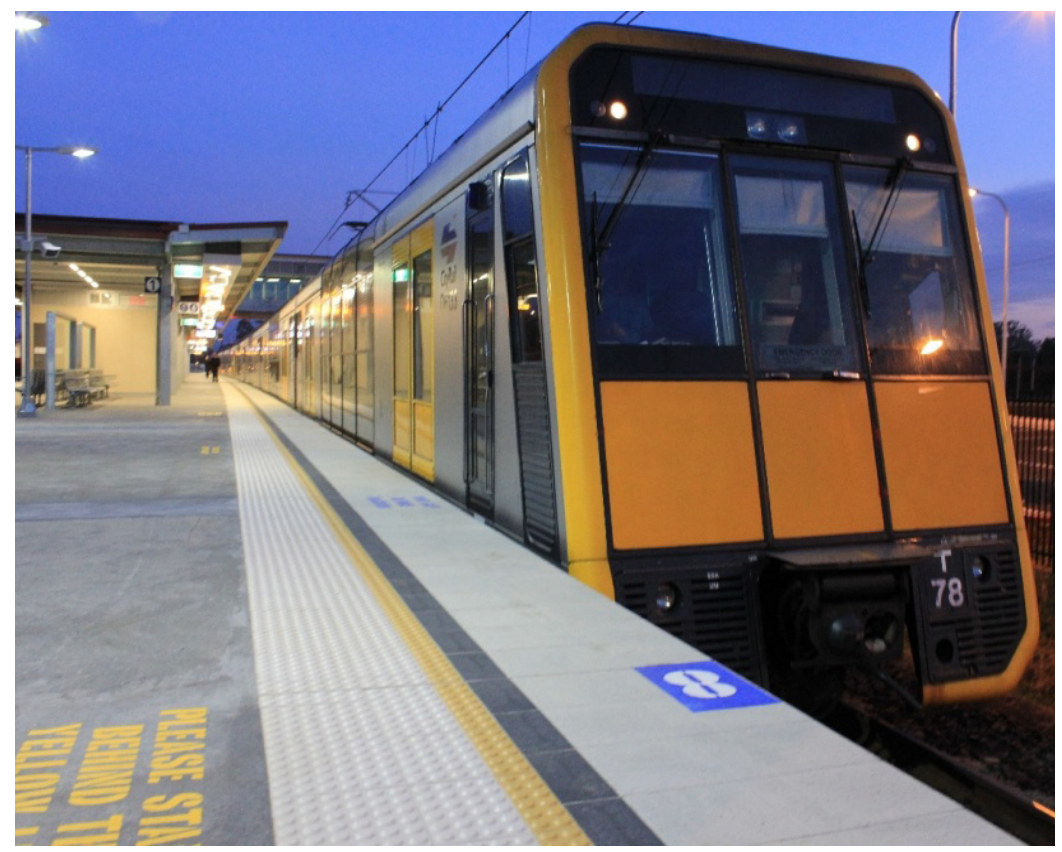

Figure 11: $\quad$ End product delivered.

\section{References}

[1] Robertson, J. Richmond Railway Line Duplication. Minister for Transport NSW Legislative Council Hansard, pp. 25522, 9 September 2010.

[2] More services for Richmond Line commuters following improvements. Transport for NSW website www.transport.nsw.gov.au/mediareleases/more-services-richmond-line-commuters-following-improvements

[3] Project Alliance Agreement Quakers Hill to Vineyard Duplication. Transport forNSW website. www.transport.nsw.gov.au/projects/projecttoolkit/register-of-contracts-awarded

[4] Smith, M and Boulden, P. Richmond Line Alliance - A Case Study in Effective Project Development and Delivery of a Complete Rail System. Proc. of CORE2010 Conference of Railway Engineering: Wellington, NZ, pp. 663-668, 2010.

[5] Welschen, R. Risk Management Innovations on a Construction Project. NSW Risk Engineering Society Presentation, Engineers Australia, 29 October 2009. 